\title{
The generalized risk scale - a scalar integrated tool for developing risk criteria by consensus, in the field of explosives for civil uses
}

\author{
Camelia Lavinia Unguras ${ }^{1 *}$, Doru Anghelache ${ }^{1}$, Victor Gabriel Vasilescu ${ }^{1}$, Florian Stoian ${ }^{1}$, \\ and Gabriel Ioan Ilcea² \\ ${ }^{1}$ University of Petrosani, Doctoral School, 20 University St., Petrosani, Romania \\ ${ }^{2}$ University of Petrosani, Research Department, 20 University St., Petrosani, Romania.
}

\begin{abstract}
The paper highlights the result of the critical analysis upon criteria regarding the safety of explosives based on the quantification of risks specific for handling explosive materials, for the proper criteria selection and for defining the integrated concept "how safe is safe enough?", applicable to complex work systems whose activity object involves the use of hazardous substances such as explosives for civil use. In this regard are conceptual and applicative presented judicial precedents and standards which are used for establishing risk acceptance criteria. These data and information are represented graphically along a series of logarithmic scales which ensure an objective manner for risk quantification based on scientific reasoning, real information derived from a data base which is specific for a deep knowledge of morbidity indicators recorded over an a statistically acceptable.
\end{abstract}

\section{Introduction}

In 1999, the DoD (Department of Defense) sponsored the initial development of risk criteria for use in risk-based management of explosive materials. Initially, these criteria were to be used on a trial basis for decisions associated with siting of explosives facilities. To support the development of these criteria, various data relating to risk-acceptability were gathered from a variety of sources. To be compared, this data needed to be accumulated in a common format. This need led to the development of the Universal Risk Scale [1].

The Universal Risk Scale proved to be a valuable tool in reaching consensus within the Risk Based Explosives Safety Criteria Team (RBESCT) on the risk criteria used for siting explosives facilities. The scales have also been used to compare relevant data to assist policy makers in selecting appropriate risk related criteria in other areas. As the use of risk-based techniques expands within the area of explosives safety, and into other areas where hazards to the public reside, further research is needed to support the development of risk criteria applicable to these areas [2].

This paper provides an update on the RBESCT's continuing research into the fundamental question - "How safe is safe enough?"

\section{Material and method}

The logarithmic scale is preferred for risk analysis because (Fig.1,2):

- $\quad$ Range of interest for risk spans many orders of magnitude;

${ }^{1}$ Corresponding author: camelialaviniaunguras@gmail.com 
- Proportional logic - Multiplying the risk by a constant factor gives a constant separation;

- In widespread use for quantitative risk assessment.

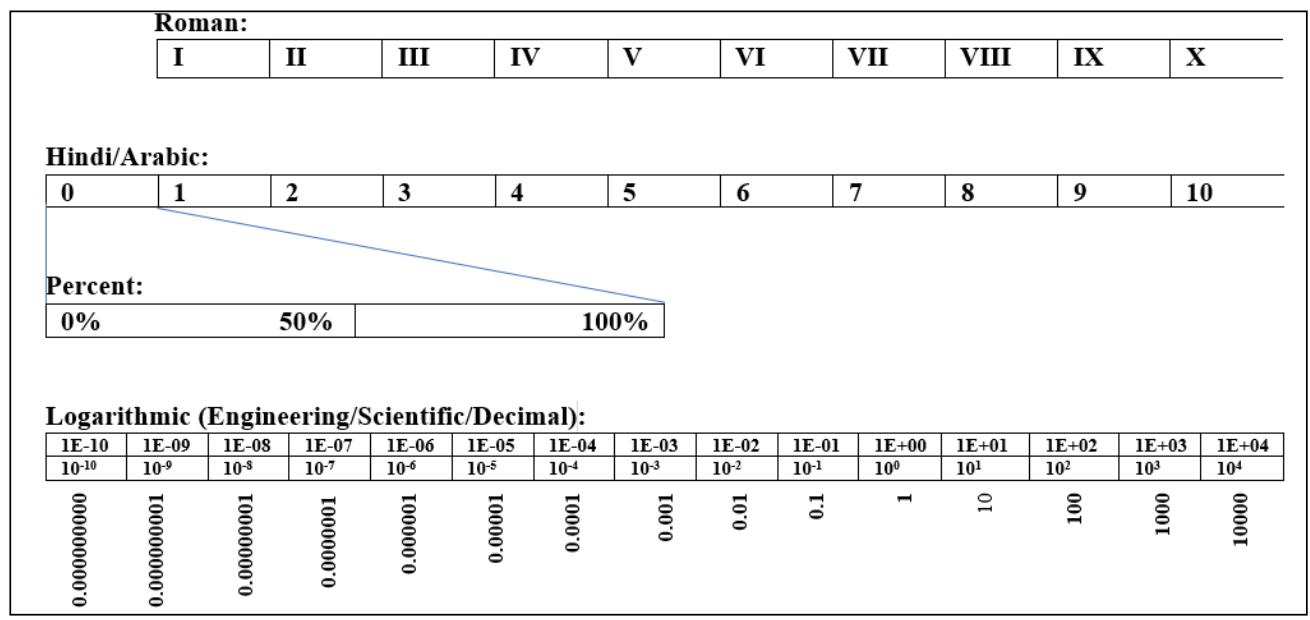

Fig.1 Number scales with logarithmic values represented in engineering notation

\begin{tabular}{|l|c|c|c|}
\hline & Fraction & $\begin{array}{c}\text { Scientific } \\
\text { notation }\end{array}$ & $\begin{array}{c}\text { Engineering } \\
\text { notation }\end{array}$ \\
\hline \multirow{2}{*}{ Fraction } & $2 / 3$ & $6.67 \times 10^{-1}$ & $6.67 \mathrm{E}-01$ \\
\hline \multirow{2}{*}{ Small fraction } & $1 / 10$ & $1.00 \times 10^{-1}$ & $1.00 \mathrm{E}-01$ \\
\cline { 2 - 4 } & $1 / 3,000$ & $3.33 \times 10^{-4}$ & $3.33 \mathrm{E}-04$ \\
\hline \multirow{2}{*}{$\begin{array}{c}\text { Extremely small } \\
\text { fraction }\end{array}$} & $1 / 100,000$ & $7.00 \times 10^{-5}$ & $7.00 \mathrm{E}-05$ \\
\cline { 2 - 4 } & $5 / 10,000,000,000$ & $1.00 \times 10^{-8}$ & $1.00 \mathrm{E}-08$ \\
\hline
\end{tabular}

Fig.2 Number scales using the Log Scale

\subsection{Pascalian Methods}

According to the Pascalian Methods (Fig.3), [3]: Concept of risk: Risk = Likelihood $x$ Consequence; Proportionality: Consistency in risk space; Systematic devolution: Description of contributing elements; Preciseness: Quantitative.

\begin{tabular}{|l|c|c|c|c|}
\hline $\begin{array}{c}\text { Mishap } \\
\text { Probability } \\
\text { Levels }\end{array}$ & \multicolumn{4}{|c|}{ Mishap Severity Categories } \\
\cline { 2 - 5 } & (1) Catastrophic & (2) Critical & (3) Marginal & (4) Negligible \\
\hline (A) Frequent & High & High & Serious & Medium \\
\hline (B) Probable & High & High & Serious & Medium \\
\hline (C) Occasional & High & Serious & Medium & Low \\
\hline (D) Remote & Serious & Medium & Medium & Low \\
\hline (E) Improbable & Medium & Medium & Medium & Low \\
\hline
\end{tabular}

Fig.3 Example Risk Assessment Matrix as viewed by Pascal's Proportional Concept 
According to the Risk Assessment Methods (Fig.4), [3]:

- Qualitative: Risk assessment are often subjective and based on judgments

- Quantitative: Risk assessment are based on the best available information including accident history, physical science, test results, expert judgment and statistics.

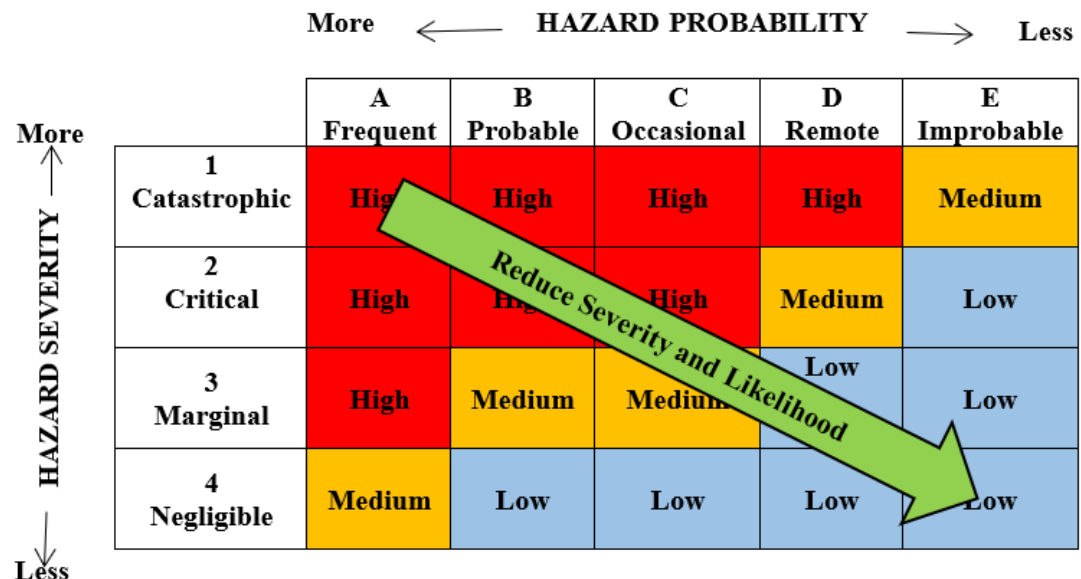

Fig.4 Risk Assessment Matrix

Basic risk equation:

$$
\text { Risk }=\text { Probability } \mathrm{x} \text { Consequence }
$$

Basic risk equation to person(s):

$$
\text { Risk }=\text { Probability } \mathrm{x} \text { (Consequence level } \mathrm{x} \text { Human Exposure) }
$$

Equation of risk to person(s) from explosives events - Annual risk:

$$
\mathrm{P}_{\mathrm{f}}=\mathrm{P}_{\mathrm{e}} \times \mathrm{P}_{\mathrm{f} / \mathrm{e}} \times \mathrm{E}_{\mathrm{p}}
$$

Where: $\mathrm{P}_{\mathrm{f}}$-Probability of fatality; $\mathrm{P}_{\mathrm{e}}$-Probability of event; $\mathrm{P}_{\mathrm{f} / \mathrm{e}}$-Probability of fatality given an event and a person; $\mathrm{E}_{\mathrm{p}}$-Exposure hours per year

Expansion of $\mathrm{P}_{\mathrm{f} / \mathrm{e}}$ term: Pressure/Impulse, Glass and Building Failure, Debris, Temperature

\subsection{Criteria Basis}

Basis of Criteria for "Safe Enough" is showing in Fig.5. 


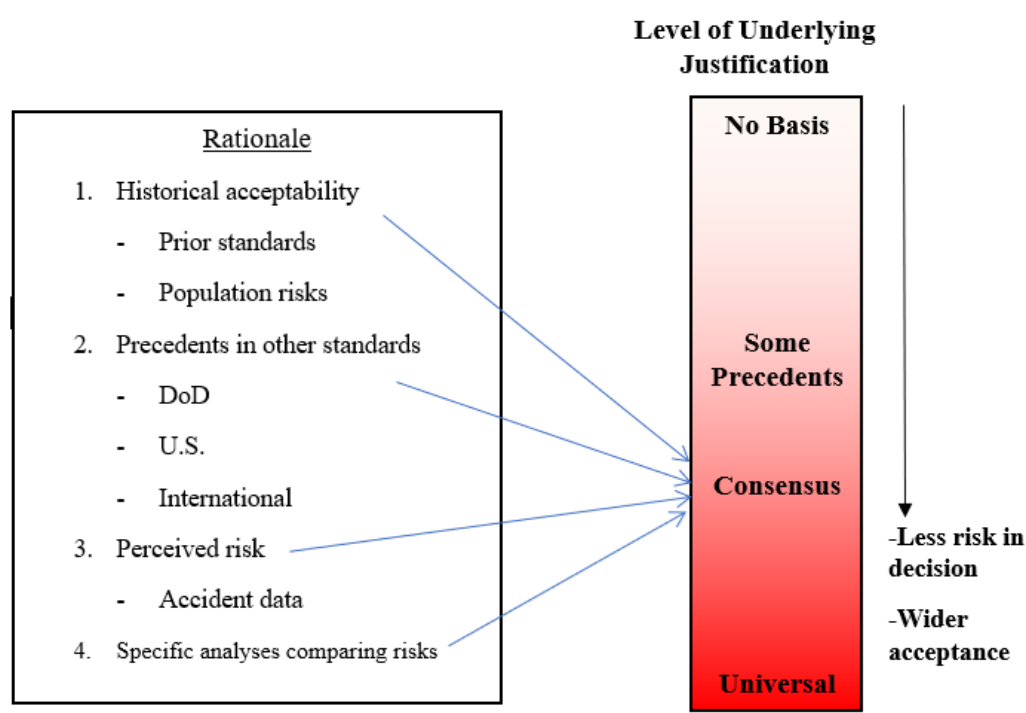

Fig.5 Criteria Basis

\section{Results and Discussion}

\subsection{The Universal Risk Scale Format}

The answer to this question, "How safe is safe enough?" is an essential ingredient in establishing any risk criterion. Though the question is fundamental to achieving the practical goal of establishing risk criteria, it is also a somewhat philosophical question, in that it requires individuals to make subjective interpretations of legal precedents, societal values and past risk experiences [3].

Opinions vary widely as to what types of information should be considered when making these judgments, and these differences of opinion become all the more pronounced when the relative importance of individual data points is considered. For this reason, consensus decisions regarding risk criteria are particularly difficult to achieve. To facilitate decisions of this type, the Universal Risk Scale was developed to display on a single scale a wide variety of information for the purpose of comparison. The intent is to display as much information as practical, with the hope that the individual participants in the decision will find among the data, information they consider relevant.

There are two primary types of information shown on the Universal Risk Scale. The first, is various risk-related legal precedents and governmental standards which may be considered relevant to the case at hand, the second is real-world statistical data derived from documented accident experience (Fig.6). 


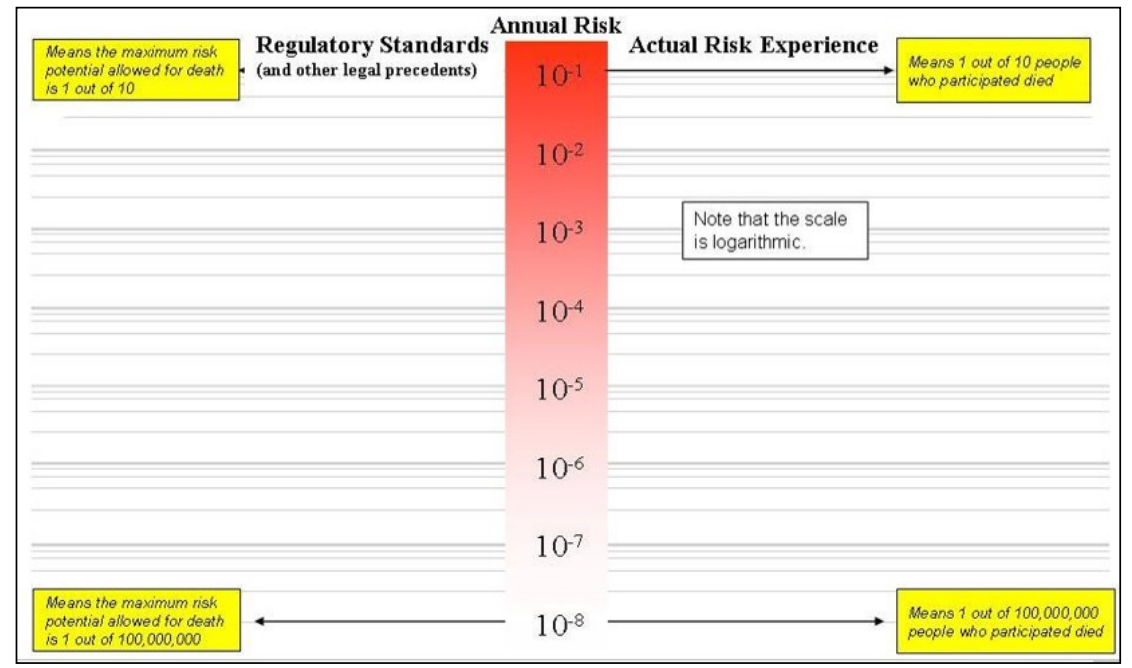

Fig.6 Universal Risk Scale Format

Figure 6 shows the format of the Universal Risk Scale. The logarithmic scale was chosen because it can display a wide variety of disparate data and allows the aggregate weight of the individual data points to be viewed at once. This scale also enables large differences in the amount of actual risk to be displayed in a small numerical space.

For instance, the difference between the values of zero and one on a linear scale is small; in fact, most people think of this numerical space in linear terms of percent. The linear paradigm, however, does not provide the necessary perspective for a useful understanding of the concept of risk. Measured risk is better viewed logarithmically; as orders of magnitude, to allow comparisons of relative risk.

The Universal Risk Scale format attempts to achieve this perspective so that the concept of relative risk can be more properly understood.

\subsection{The Two Universal Risk Scales}

In the figures that follow, all data are shown in terms of annual risk. The surrounding data points are the product of research for relevant supporting data. Many data points are shown because individuals may ascribe more or less relevance to each data point [4].

\subsubsection{Risk to Any One Worker}

The scale supporting the criterion for protection of any one worker is shown in Figure 7 [5,610]. This scale is labeled "individual voluntary" because the risk to the individual is accepted voluntarily as a condition of employment. Figure 7 plots the data on a Universal Risk Scale and the following paragraphs describe each data point [11,12-15].

The reactions still continue in the Taylor rarefaction wave, between the sonic line and end of reaction zone, and they cannot contribute to the shock front but they can significantly contribute to the blast. 


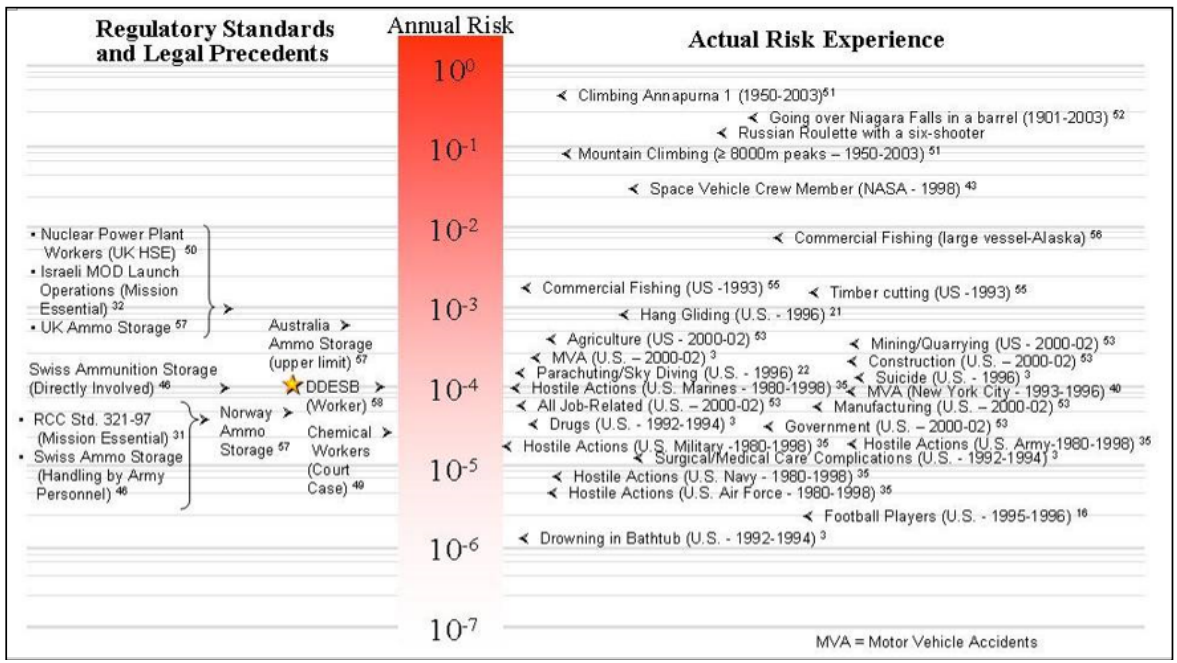

Fig.7 Scale - Individual Voluntary

\subsubsection{Risk to Any One Person}

The scale supporting the protection criterion for any one person is shown in Figure 8. This scale is labeled "individual involuntary" because the risk is not accepted as a voluntary action taken by an individual. For example, victims of homicide, stroke or tornado generally do not die as the result of a voluntary decision to accept risk. Figure 8 plots the data on a Universal Risk Scale and the following paragraphs describe each data point [11,12-15].

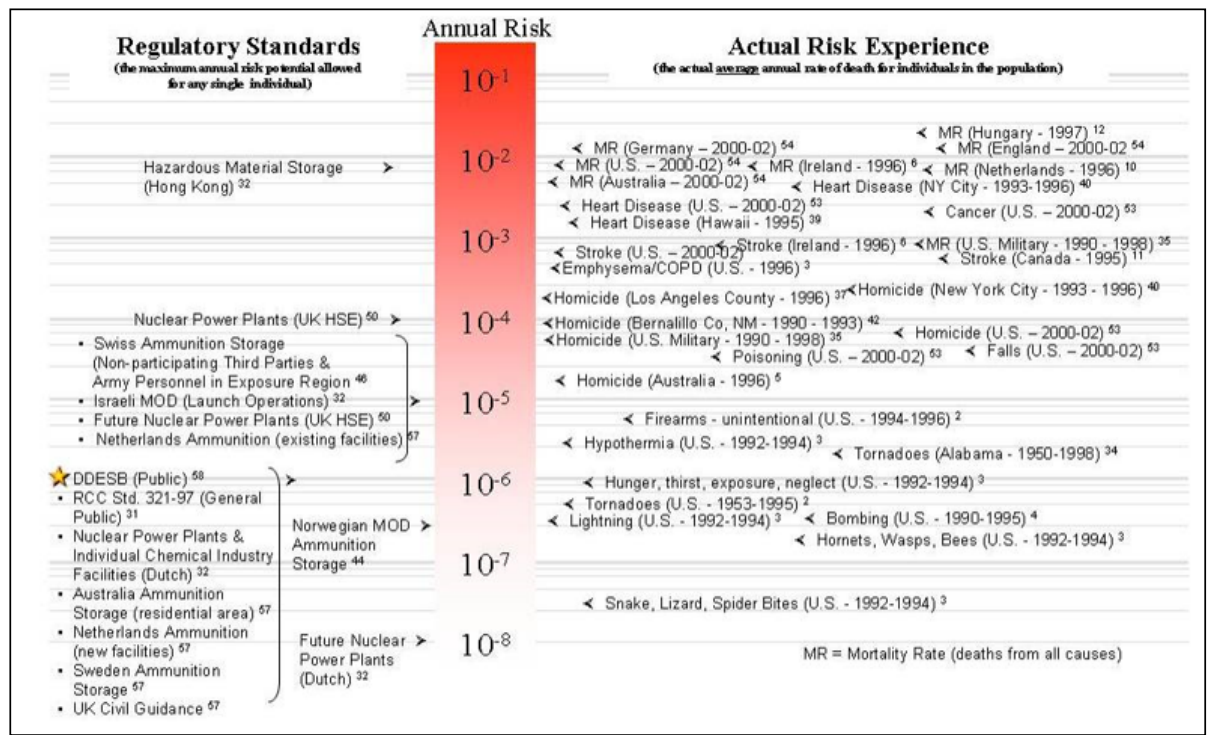

Fig.8 Scale - Individual Involuntary 


\section{Conclusions}

The RBESCT (Risk Based Explosives Safety Criteria Team) has been conducting research for data to support the criteria chosen for personnel protection.

Accident data, regulations, and legal precedents have been reviewed to identify data relevant to the level of personnel protection. These data have been plotted on the Universal Risk Scales.

A foundation has been laid that can benefit the international explosives safety community, as well as other safety communities who are using risk-based analyses and numerical risk criteria.

\section{References}

1. DoD Washington Headquarters Services - Directorate for Information Operations and Reports. "Department of Defense - Military Casualty Information." http://web1.whs.osd.mil December 1998 (13 April 1999).

2. Swisdak, Michael, "DDESB Blast Effects Computer Version 5 User's Manual and Documentation," DDESB Technical Paper 17, 10 March 2005.

3. National Center for Health Statistics. "Vital and Health Statistics of the United States." http://www.cdc.gov/nchswww October 1998 (5 January 1999).

4. "RBESCT Technical Issues Regarding Injury Modeling," APT CE3-00300, 26 February 2004.

5. U.S. Commissioner of Pensions Annual Report. "Department of Defense - Historical Background and Notes." http://web1.whs.osd.mil/mmid/m01/sms223rr.htm December 1998 (13 April 1999).

6. California Department of Justice, Criminal Justice Statistics Center. "California Crime Index, 1996." http://www.caag.state.ca.us/cjsc/datatabs.htm March 1999 (13 April 1999).

7. District of Columbia Department of Health State Center for Health Statistics. "Vital Statistics Data Sheet 1995." http://www.dchealth.com/preview/schs/reports.stm September 1996 (13 April 1999).

8. Bureau of Mines I.C. 7493, Bulletin 586; Major Disasters at Metal and nonmetal Mines and Quarries in the U.S. (Excluding Coal Mines).

9. National Center for Health Statistics. National Health Interview Survey, 1994. Vital and Health Statistics. Item 10 (93).

10. National Safety Council. 1997. Accident Facts - 1997 Edition. Itasca, IL: NSC.

11. FBI Explosives Unit Bomb Data Center. "Annual Bombing Statistics in the United States." http://www.fbi.gov/lab/bomsum/eubdc.htm August 1998 (21 December 1998).

12. IMESAFR P(e) Matrix Tech Memo, APT Report CM-07400, May 2006.

13. Central Statistics Office of Ireland. "Principle causes of death, crude death rates and age-standardised mortality rates." http://www.doh.ie/stats/b07.htm 17 December 1998 (22 December 1998).

14. Federal Statistical Office Germany. "Population development and life expectancy." http://www.statistik-bund.de/basis/e July 1998 (22 December 1998).

15. Instituto Nacional de Estadistica. "Number of deaths by major causes of death." http://www.ine.es/htdocs/dacoin/dacoinci/sanitari August 1998 (22 December 1998). 